\title{
AKT (protein kinase B) is implicated in meiotic maturation of porcine oocytes
}

\author{
Jaroslav Kalous, Michal Kubelka, Petr Šolc, Andrej Šušor and Jan Motlík \\ Institute of Animal Physiology and Genetics, Academy of Sciences of the Czech Republic, Rumburska 89, 27721 \\ Libechov, Czech Republic \\ Correspondence should be addressed to J Kalous; Email: kalous@iapg.cas.cz
}

\begin{abstract}
The aim of this study was to investigate the involvement of the serine/threonine protein kinase AKT (also called protein kinase B) in the control of meiosis of porcine denuded oocytes (DOs) matured in vitro. Western blot analysis revealed that the two principal AKT phosphorylation sites, Ser473 and Thr308, are phosphorylated at different stages of meiosis. In freshly isolated germinal vesicle (GV)-stage DOs, Ser473 was already phosphorylated. After the onset of oocyte maturation, the intensity of the Ser473 phosphorylation increased, however, which declined sharply when DOs underwent GV breakdown (GVBD) and remained at low levels in metaphase I- and II-stage (MI- and MII-stage). In contrast, phosphorylation of Thr308 was increased by the time of GVBD and reached maximum at MI-stage. A peak of AKT activity was noticed around GVBD and activity of AKT declined at MI-stage. To assess the role of AKT during meiosis, porcine DOs were cultured in $50 \mu \mathrm{M}$ SH-6, a specific inhibitor of AKT. In SH-6-treated DOs, GVBD was not inhibited; on the contrary, a significant acceleration of meiosis resumption was observed. The dynamics of the Ser473 phosphorylation was not affected; however, phosphorylation of Thr308 was reduced, AKT activity was diminished at the time of GVBD, and meiotic progression was arrested in early MI-stage. Moreover, the activity of the cyclin-dependent kinase 1 (CDK1) and MAP kinase declined when SH-6-treated DOs underwent GVBD, indicating that AKT activity is involved in the regulation of CDK1 and MAP kinase. These results suggest that activity of AKT is not essential for induction of GVBD in porcine oocytes but plays a substantial role during progression of meiosis to MI/MII-stage.

Reproduction (2009) 138 645-654
\end{abstract}

\section{Introduction}

In mammalian ovaries, the oocytes are naturally arrested in prophase of the first meiotic division, at the so-called germinal vesicle (GV) stage. The fully grown oocytes resume meiosis in vivo after $\mathrm{LH}$ surge or in vitro when released from antral follicles and further cultured under suitable conditions.

cAMP is one of the main regulators of the oocyte entry into meiosis. In mammalian oocytes, the concentration of cAMP is regulated by gonadotropins such as FSH and LH acting through the follicular cells (Bornslaeger \& Schultz 1985). In mouse oocytes, elevated levels of cAMP and activated protein kinase $\mathrm{A}$ (PKA) are involved in maintaining oocytes in GV stage (Tsafriri et al. 1996, Conti et al. 1998). PKA activity is maintained at high levels by the increased CAMP, thus preventing maturation in vitro and in vivo (Masciarelli et al. 2004). Degradation of cAMP by a phosphodiesterase type IIIA (PDE3A) leads to a decrease of PKA activity and initiates resumption of meiosis in rodent oocytes (Conti 2002, Kovo et al. 2006). Similarly, in porcine oocytes, resumption of meiosis is also associated with a decrease in CAMP concentration and subsequent inactivation of PKA (Kim \& Menino 1995).
The activity of maturation promoting factor (MPF) is crucial for meiotic maturation of oocytes. MPF complex consists of the catalytic subunit p34 cdc2 kinase (also termed cyclin-dependent kinase 1 (CDK1)) and regulatory subunit cyclin B (Gautier \& Maller 1991, Jessus \& Beach 1992). The activity of CDK1 is controlled by phosphorylation at three residues; phosphorylation on Thr161 is necessary for CDK1 kinase activation, whereas phosphorylation of CDK1 by WEE1 and MYT1 kinases on either Thr14 or Tyr15 residues dominantly inhibits activation of CDK1 (Coleman \& Dunphy 1994). This inhibition is reversed when CDK1 is dephosphorylated by activated CDC25C phosphatase (Nilsson \& Hoffmann 2000). It has been reported that PKA phosphorylates and inactivates CDC25C in frog oocytes (Duckworth et al. 2002, Shibuya 2003). Also in mouse oocytes, the WEE1 kinase is a substrate for PKA in vitro (Han et al. 2005). These findings support the concept that PKA maintains meiotic arrest via dual regulation of the kinase that phosphorylates CDK1 and the phosphatase that dephosphorylates the same residues of CDK1 (Han \& Conti 2006). 
Another kinase implicated in MPF regulation is a serine/threonine protein kinase B (PKB/AKT), a key downstream effector of the phosphatidylinositol 3-kinase (PI3K) pathway.

The PI3K-AKT pathway is activated by insulin, growth factors, and adhesion to extracellular matrix or other cells. Activated PI3K converts its lipid substrate phosphatidylinositol 4,5 biphosphate (Ptdlns $(4,5) \mathrm{P}_{2}$ ) to phosphatidylinositol $3,4,5$ triphosphate (Ptdlns $\left.(3,4,5) \mathrm{P}_{3}\right)$. Consequently, AKT is recruited to the cell membrane after binding PtdIns $(3,4,5) \mathrm{P}_{3}$ through its $\mathrm{PH}$ domain, and membrane-bound AKT is activated by phosphorylation at two sites. PDK1 phosphorylates Thr308 residue located in the activation loop of the kinase (Stokoe et al. 1997, Kim et al. 2001), whereas mTORC2 was identified as the kinase responsible for phosphorylation of the Ser473 residue situated in the hydrophobic site (Sarbassov et al. 2005). The dualspecificity lipid phosphatase phosphatase and tensin homolog deleted on chromosome 10 (PTEN) dephosphorylates $\mathrm{PI}(3,4,5) \mathrm{P}_{3}$ and in such a way negatively regulates AKT kinase activity (Maehama et al. 2001).

It has been reported that PI3K/AKT pathway contributes to cyclin B1 expression and CDK1 activation (Roberts et al. 2002). Data obtained on somatic cells indicate that AKT promotes cell survival and participates in the regulation of the G2/M transition. In epithelial cells, the activity of AKT is increased at the G2/M transition of the cell cycle, and it is necessary for the timely progression through mitosis (Shtivelman et al. 2002). The cell cycle arrest imposed by inhibitors of PI3K could be reversed by the expression of constitutively active AKT (Shtivelman 2003). A G2/M cell cycle checkpoint induced by DNA damage is surpassed by constitutively activated AKT, and this effect has also been observed in cells lacking PTEN that limits activation of AKT (Kandel et al. 2002). AKT was also reported to promote cell cycle progression at the G2/M transition through WEE1 inactivation in HEK 293 cells (Katayama et al. 2005). In HeLa cells, AKT phosphorylates the CDC25B phosphatase and regulates its intracellular localization (Baldin et al. 2003).

The glycogen synthase kinase-3 (GSK3), an important substrate of AKT, is involved in numerous cellular processes, including regulation of the activity of microtubule-associated proteins affecting formation and function of mitotic spindle (Moreno \& Avila 1998). In mouse oocytes, pharmacological inhibition of GSK3 causes abnormal meiotic spindle configuration, chromatin organization, and bivalent chromatin segregation (Wang et al. 2003).

Experiments performed on starfish oocytes provided the evidence of AKT as an initiator of the meiotic $M$ phase. It has been documented that AKT activates meiotic G2/M transition by phosphorylation and inactivation of protein kinase MYT1, which has been shown to phosphorylate and inactivate MPF
(Okumura et al. 2002). Study on Xenopus oocytes revealed that insulin-like growth factor 1 (IGF1)- or insulin-induced meiotic maturation is mediated by activation of PI3K and AKT (Liu et al. 1995, DeuterReinhardt et al. 1997). It has been shown that expression of constitutive active $\mathrm{PI} 3 \mathrm{~K}$ can activate MAPK and induce GV breakdown (GVBD) in Xenopus oocytes, and this effect is mediated through its lipid kinase activity and activation of AKT (Hehl et al. 2001). Furthermore, it was documented in Xenopus oocytes that phosphorylation of PDE3A by AKT plays a role in meiotic maturation induced by growth factors (Andersen et al. 2003). Also the data obtained in mouse oocytes indicate that activation of PDE3A by PKB-mediated phosphorylation is implicated in the control of PDE3A activity and resumption of meiosis (Han et al. 2006). Suppression of AKT activity was accompanied with a decrease of CDK1 activity and postponed resumption of meiosis in mouse oocytes (Kalous et al. 2006). Similarly, in cattle oocytes, AKT is also involved in the regulation of meiotic metaphase I and II (MI/MII) transition (Tomek \& Smiljakovic 2005).

The objectives of this study were to evaluate the dynamics of AKT phosphorylation and activation in porcine oocytes matured in vitro and to analyze possible role of AKT in the regulation of meiosis.

\section{Results \\ Over $80 \%$ of porcine denuded oocytes reached MII-stage during control culture}

In experiment 1, fresh porcine oocyte-cumulus complexes (OCCs) were deprived of cumulus, and denuded oocytes (DOs) were cultured in control medium subsequently. During $44 \mathrm{~h}$ of control culture, $84 \%$ of DOs reached MII-stage (Fig. 1). However, at $24 \mathrm{~h}$ culture interval, the time course of meiotic maturation was not synchronous as $18 \%$ of DOs retained GV, $27 \%$ exhibited late diakinesis stage (LD), 36\% advanced to $\mathrm{MI}$, and $19 \%$ to Mll-stage (Fig. 1).

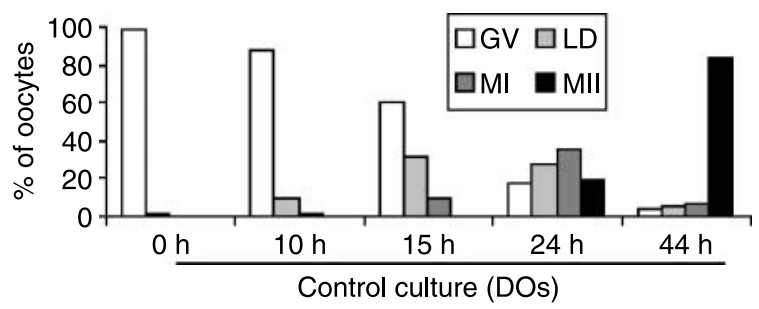

Figure 1 Progression of meiosis in porcine DOs cultured in control conditions. Isolated porcine OCCs were deprived of cumulus cells, and DOs oocytes were subsequently cultured in vitro in control medium. GV, germinal vesicle; LD, late diakinesis; MI, metaphase-I; MII, metaphase-II. A total of 977 oocytes were scored. 
A

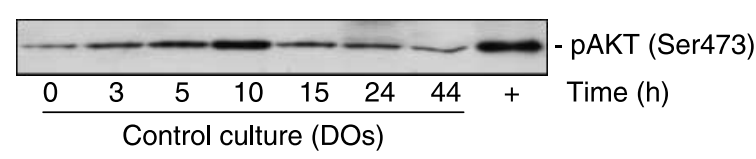

B
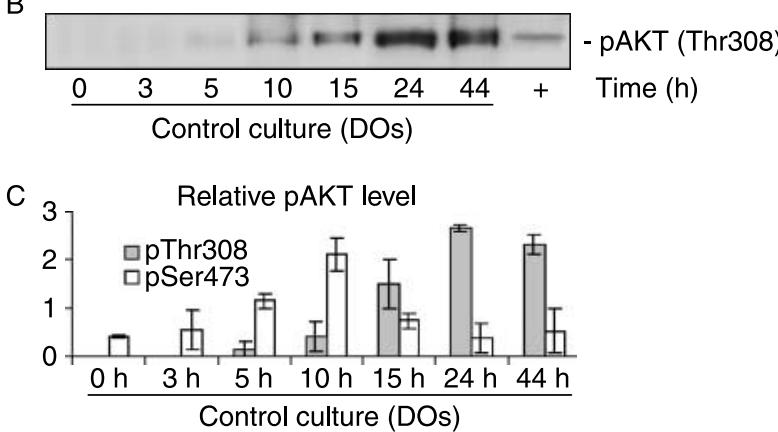

Figure 2 Phosphorylation of AKT in porcine DOs cultured in control conditions. Isolated porcine OCCs were deprived of cumulus cells, and DOs were subsequently cultured in vitro in control medium for indicated times. (A) Western blot with anti-pSer473 antibody. Lysates of $50 \mathrm{DOs} / \mathrm{sample}$ were evaluated. Extracts of PDGF-treated NIH 3T3 cells were used as a positive control (+). (B) Western blot with antipThr308 antibody. Lysates of $100 \mathrm{DOs} /$ sample were evaluated. Extracts of PDGF-treated NIH 3T3 cells were used as a positive control (+). (C) Bar graph of pSer473 and pThr308 levels. Each bar represents the mean \pm s.D. ( $n=3$ for each time interval).

\section{AKT is phosphorylated and activated during in vitro control culture of porcine DOs}

In experiment 1, Ser473 was already phosphorylated when freshly isolated OCCs were stripped of cumulus, and noncultured DOs were subjected to immunoblot analysis (Fig. 2A and C). When DOs were further cultured in control conditions, the intensity of Ser473 phosphorylation increased initially, dropped around GVBD at $15 \mathrm{~h}$ of culture, and remained at low levels at 24 and $44 \mathrm{~h}$ culture interval (Fig. 2A and C). Phosphorylation of Thr308 was not detected in fresh DOs, but emerged when DOs were cultured in control and peaked

A
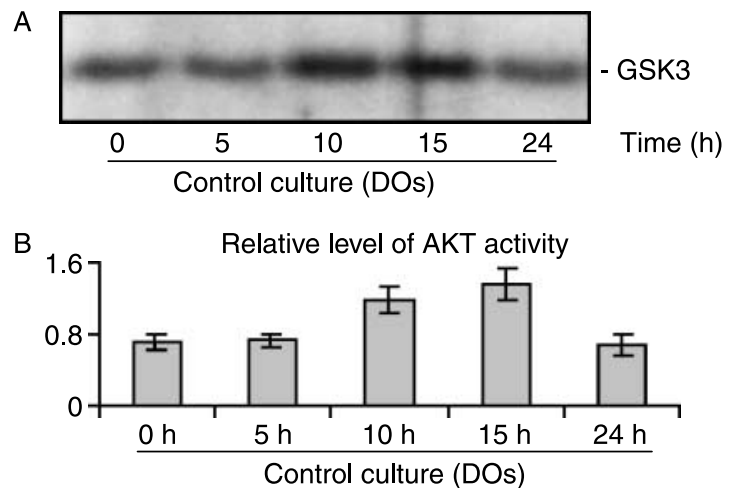

Figure 3 AKT kinase activity in porcine DOs cultured in control conditions. (A) AKT kinase assay (immunoprecipitates of 200 oocytes/sample were assayed). (B) Bar graph of relative AKT activity. Each bar represents the mean \pm s.D. ( $n=3$ for each time interval).

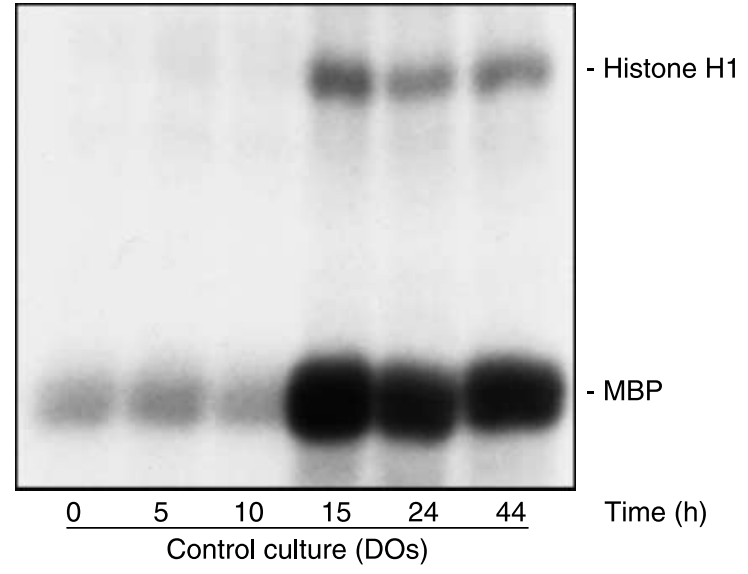

Figure 4 CDK1 and MAP kinase activities in porcine DOs cultured in control conditions. Kinase assay was performed on lysates of ten oocytes/sample.

at $24 \mathrm{~h}$ culture interval (Fig. 2B and C). The activity of AKT, being low in fresh uncultured DOs, increased during GVBD occurring at 10 and $15 \mathrm{~h}$ of culture and declined subsequently (Fig. 3A and B). The CDK1 and MAP kinase activities reached its maximum at $15 \mathrm{~h}$ of culture, and the activities of both kinases remained elevated until the $44 \mathrm{~h}$ culture interval (Fig. 4).

\section{Dibutyryl cAMP pretreatment resulted in a synchronized progression of meiosis}

In experiment 2, OCCs were initially pretreated with $1 \mathrm{mM}$ dibutyryl cAMP (dbcAMP) for $20 \mathrm{~h}$ and stripped of cumulus subsequently. When DOs were further cultured in control medium, $44 \%$ of DOs progressed to Ml-stage and $40 \%$ to Mll-stage at $24 \mathrm{~h}$ culture interval (Fig. 5). These data indicate that the dbcAMP pretreatment resulted in a more synchronous progression of meiosis comparing with the dbcAMP-nontreated oocytes in experiment 1 (Fig. 1).

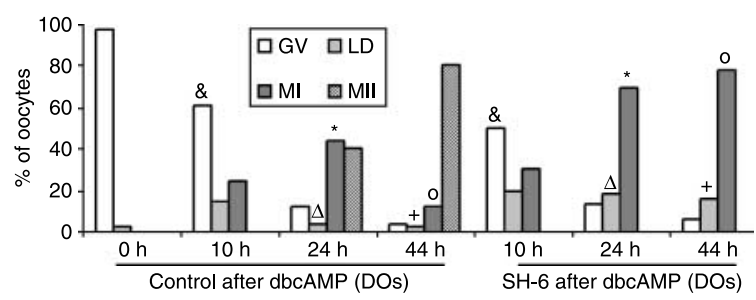

Figure 5 Progression of meiosis in dbcAMP-pretreated porcine oocytes cultured either in control or SH-6-supplemented medium. Isolated porcine OCCs were pretreated with $1 \mathrm{mM}$ dbcAMP for $20 \mathrm{~h}$, deprived of cumulus subsequently, and DOs were further cultured in vitro either in control or SH-6 (50 $\mu \mathrm{M})$-supplemented medium for indicated time intervals. Bars with identical superscripts represent significantly different data $(P<0.05)$. GV, germinal vesicle; LD, late diakinesis; MI, metaphase-I; MII, metaphase-II. A total of 1657 oocytes were scored. 


\section{Inhibition of AKT impairs progression of meiosis}

The data from experiment 2 reveal that GVBD was not inhibited in DOs cultured in $50 \mu \mathrm{M} \mathrm{SH}-6$. On the contrary, SH-6 treatment significantly $(P<0.05)$ promoted resumption of meiosis as $50 \%$ of $\mathrm{SH}-6$-treated DOs and 39\% of control DOs underwent GVBD within $10 \mathrm{~h}$ of culture (Fig. 5). These data suggest that AKT inhibitor $\mathrm{SH}-6$ has possible side effect upon regulatory mechanism of meiotic resumption. Moreover, in $\mathrm{SH}$ 6-treated DOs, progression of meiosis was arrested in early $\mathrm{Ml}$-stage. At $24 \mathrm{~h}$ of culture, $44 \%$ of control dbcAMP-pretreated oocytes reached MI-stage and exhibited regular MI-spindle (Figs 5 and $6 \mathrm{~A}$ ). At this time interval, in all of the $69 \%$ of $\mathrm{Ml}$-stage $\mathrm{SH}-6$ treated oocytes, an aberrant $\mathrm{Ml}$-spindle was observed (Figs 5 and 6B). These data indicate that inhibition of AKT interfered with formation of the regular MI-spindle and progression of meiosis to Mll-stage. There was no difference in MI-spindle conformation when both dbcAMP-pretreated and nontreated oocytes were cultured in control conditions.

\section{Kinase activity is restored when dbcAMP-pretreated oocytes are released to control culture}

In experiment 2, phosphorylation of Ser473 decreased (Fig. 7), phosphorylation of Thr308 was low (Fig. 8), and AKT activity declined (Fig. 9) in oocytes pretreated with dbcAMP for $20 \mathrm{~h}$. Activity of CDK1 and MAP kinase did not increase as well (Fig. 10). After the dbcAMPpretreated OCCs were deprived of cumulus and DOs were further cultured in control medium, meiosis resumed and phosphorylation of Ser473 increased rapidly and remained elevated until $44 \mathrm{~h}$ of culture
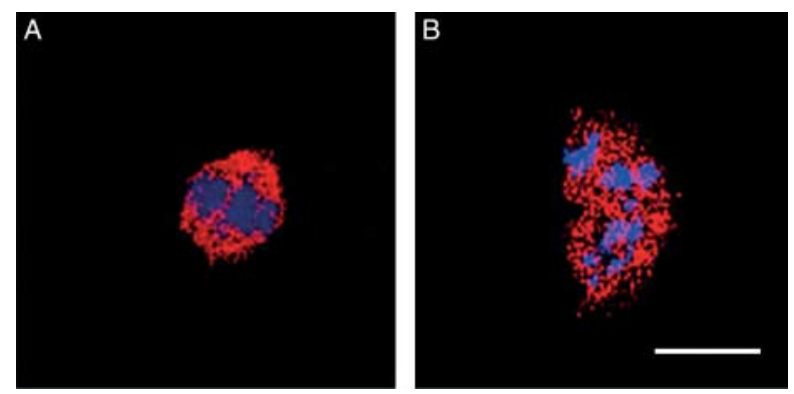

Figure 6 Representative confocal images of porcine oocytes. Porcine OCCs were precultured in $1 \mathrm{mM}$ dbcAMP for $20 \mathrm{~h}$, stripped of cumulus, and DOs were further cultured either in control or $\mathrm{SH}-6$ $(50 \mu \mathrm{M})$-supplemented medium for $24 \mathrm{~h}$. (A) Normal barrel-shaped $\mathrm{MI}$-spindle with regularly aligned chromosome bivalents in control oocytes cultured for $24 \mathrm{~h}$. All Ml-stage control oocytes exhibited normal barrel-shaped MI-spindle. (B) Disorganized MI-spindle with aberrantly organized chromosomes in oocytes cultured in $\mathrm{SH}-6$ $(50 \mu \mathrm{M})$-supplemented medium for $24 \mathrm{~h}$. All SH-6-treated oocytes reaching $\mathrm{MI}$-stage exhibited aberrant spindle configuration. Scale bar, $10 \mu \mathrm{m}$.
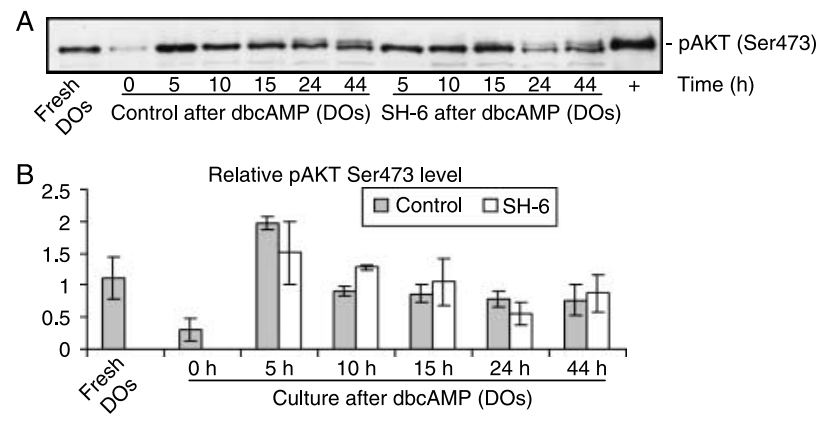

Figure 7 Phosphorylation of pSer473 AKT in dbcAMP-pretreated porcine oocytes cultured either in control or SH-6-supplemented medium. Isolated porcine OCCs were pretreated with $1 \mathrm{mM}$ dbcAMP for $20 \mathrm{~h}$, deprived of cumulus cells, and DOs were further cultured either in control or SH-6 (50 $\mu \mathrm{M})$-supplemented medium for indicated time intervals. (A) A representative western blot with anti-pSer473 antibody. Lysates of 50 oocytes/sample were evaluated. Extracts of PDGF-treated NIH 3T3 cells were used as a positive control (+). (B) Bar graph of pSer473 levels. Each bar represents the mean \pm s.D. $(n=3$ for each time interval).

(Fig. 7). Also the Thr308 residue became intensively phosphorylated reaching the maximum at $24 \mathrm{~h}$ of culture (Fig. 8). However, the quantity of AKT protein remained stable during the culture (Fig. 11). The activity of AKT increased at $10 \mathrm{~h}$ control culture interval and declined at $24 \mathrm{~h}$ (Fig. 9); the CDK1 and MAP kinase activities raised and remained elevated till the end of control culture (Fig. 10). The uniformity of protein load in analyzed samples was documented by silver staining of western blot membranes used for pSer473 and pThr308 detection (Supplementary Figure 1, which can be viewed online at www.reproduction-online.org/supplemental/).
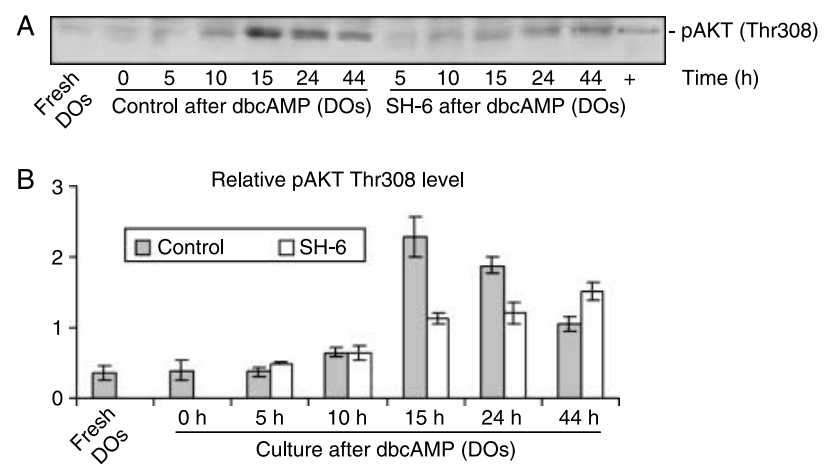

Figure 8 Phosphorylation of pThr308 AKT in dbcAMP-pretreated porcine oocytes cultured either in control or SH-6-supplemented medium. Isolated porcine OCCs were pretreated with $1 \mathrm{mM}$ dbcAMP for $20 \mathrm{~h}$, deprived of cumulus cells, and DOs were further cultured either in control or SH-6 (50 $\mu \mathrm{M})$-supplemented medium for indicated time intervals. (A) A representative western blot with anti-pThr308 antibody. Lysates of 100 oocytes/sample were evaluated. Extracts of platelet-derived growth factor-treated NIH 3T3 cells were used as a positive control (+). (B) Bar graph of pThr308 levels. Each bar represents the mean \pm s.D. ( $n=3$ for each time interval). 


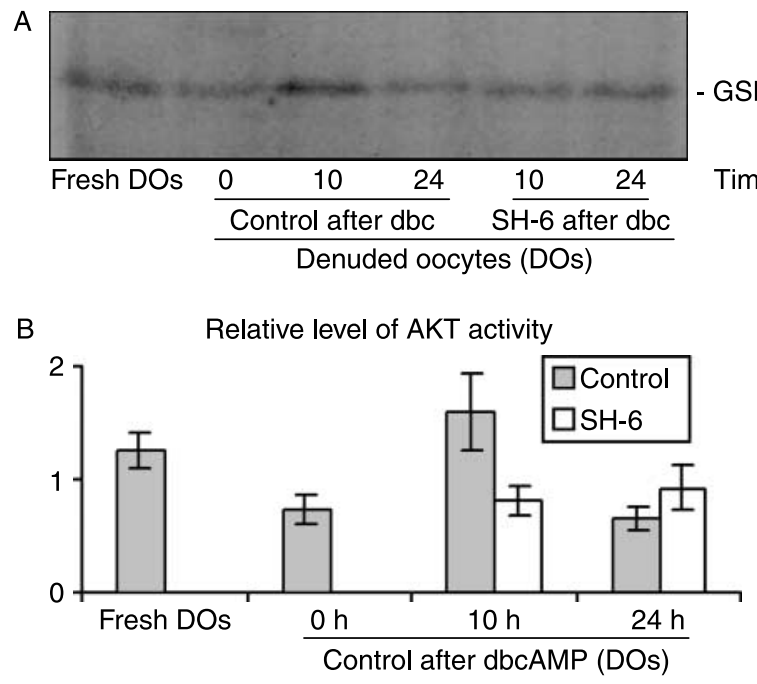

Figure 9 AKT kinase activity in dbcAMP-pretreated porcine oocytes cultured either in control or SH-6-supplemented medium. The OCCs were precultured in $1 \mathrm{mM}$ dbcAMP for $20 \mathrm{~h}$, stripped of cumulus subsequently, and DOs were further cultured either in control or SH-6 $(50 \mu \mathrm{M})$-supplemented medium for indicated time intervals. (A) AKT kinase assay (immunoprecipitates of 200 oocytes/sample). (B) Bar graph of relative AKT activity. Each bar represents the mean \pm s.D. $(n=3$ for each time interval).

\section{Inhibitor SH-6 affects phosphorylation of AKT and attenuates kinase activity}

In experiment 2, when DOs were cultured in $\mathrm{SH}$ 6-supplemented medium, AKT became phosphorylated at Thr308 with lower intensity comparing with control DOs (Fig. 8). In SH-6-treated DOs, a reduction in Thr308 phosphorylation was more prominent at $15 \mathrm{~h}$ culture interval (Fig. 8), whereas Ser473 phosphorylation was not affected (Fig. 7). AKT kinase assay revealed that $\mathrm{SH}-6$ treatment resulted in a decrease in AKT activity at $10 \mathrm{~h}$ of culture (Fig. 9). Although the initial activation of histone $\mathrm{H} 1$ kinase and MAP kinase was not prevented in $\mathrm{SH}-6$-treated DOs, suppression of activity of these kinases was apparent at 24 and $44 \mathrm{~h}$ of culture (Fig. 10).

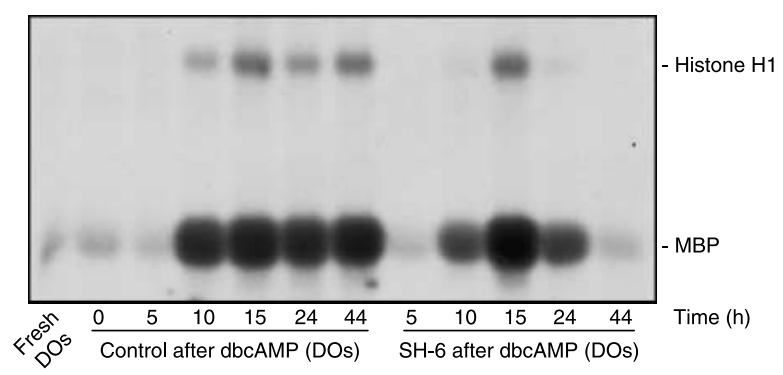

Figure 10 Activities of CDK1 and MAP kinase. Porcine OCCs were precultured in $1 \mathrm{mM} \mathrm{dbcAMP}$ for $20 \mathrm{~h}$, stripped of cumulus, and DOs were further cultured either in control or SH-6 $(50 \mu \mathrm{M})$-supplemented medium. CDK1 and MAP kinase assay was performed on lysates of ten oocytes/sample.
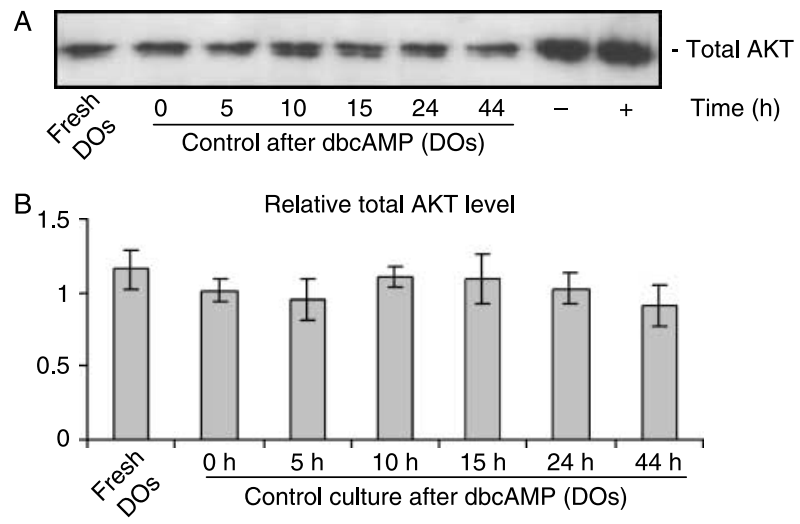

Figure 11 The levels of AKT in dbcAMP-pretreated porcine oocytes cultured in control conditions. Isolated porcine OCCs were pretreated with $1 \mathrm{mM}$ dbcAMP for $20 \mathrm{~h}$, deprived of cumulus cells subsequently, and DOs were further cultured in control conditions for indicated time intervals. (A) Western blot with total PKB antibody. Lysates of 100 oocytes/sample were evaluated. Extracts of PDGF-treated NIH 3T3 cells were used as a positive control $(+)$, whereas extracts of starved NIH 3 T3 cells were used as a negative control (-). (B) Bar graph of total AKT levels. Each bar represents the mean \pm s.D. $(n=3$ for each time interval).

\section{Discussion}

As we previously reported, AKT is involved in CDK1 activation and resumption of meiosis in mouse oocytes (Kalous et al. 2006). In porcine OCCs, the activity of AKT is involved in cumulus expansion by promoting the $\mathrm{FSH}$ stimulated synthesis of hyaluronic acid (Nemcova et al. 2007). However, when porcine OCCs are cultured in gonadotropin-free medium, expansion of cumulus layer does not occur, AKT in oocytes is dephosphorylated, and GV in oocytes persists for $24 \mathrm{~h}$ (J Kalous, M Kubelka \& J Motlík 2007, unpublished results). In order to achieve meiotic resumption of porcine oocytes in vitro without affecting AKT pathway by treatment of OCCs by gonadotropins, the present study was performed on oocytes deprived of cumulus. In experiment 1, we documented that AKT is phosphorylated and activated during progression of meiosis in porcine dbcAMPnontreated oocytes. In experiment 2, porcine OCCs were pretreated with dbcAMP to achieve more consistent progression of meiosis, similarly as already reported in mouse and porcine oocytes (Funahashi et al. 1997, Vanhoutte et al. 2008). After the dbcAMP-pretreated porcine OCCs were deprived of cumulus and DOs were further cultured in control conditions, phosphorylation and activity of AKT increased at the time of GVBD, similarly as revealed in experiment 1 on dbcAMPnontreated oocytes. These data are in accordance with those obtained in mouse and cattle oocytes, suggesting the importance of AKT activity during meiotic G2/M transition (Tomek \& Smiljakovic 2005, Kalous et al. 2006). Similarly, enhanced activity of AKT has been reported at the G2/M transition of somatic cells (Shtivelman et al. 2002). In experiment 2, after the 
dbcAMP-pretreated oocytes were subjected to AKT inhibitor SH-6, a reduction in Thr308 phosphorylation occurred and kinase activities of both AKT and CDK1 decreased. Moreover, meiosis in SH-6-treated DOs was arrested in early Ml-stage exhibiting defective MI-spindles. During in vitro maturation of porcine DOs, the abundance of AKT protein remained stable, similarly as reported in mouse and cattle oocytes (Tomek \& Smiljakovic 2005, Kalous et al. 2006, Hoshino \& Sato 2008).

In fresh GV-stage porcine DOs, a low activity of AKT was detected; however, Ser473 was phosphorylated in these oocytes. Likewise, phosphorylation of AKT at Ser473 has also been reported in mouse GV-intact oocytes (Smith \& Ding 2005). It has been documented that AKT-mediated phosphorylation of PDE3A plays a role in the control of PDE3A activity in mouse oocytes (Han et al. 2006). Therefore, it is possible to deduce that also in porcine GV-stage oocytes, AKT is involved in the regulation of CAMP levels. However, it has been revealed that PKB is stimulated by various stress factors including $\mathrm{H}_{2} \mathrm{O}_{2}$ (Konishi et al. 1997). This suggests that Ser473 phosphorylation and partial activation of AKT in fresh GV-stage porcine oocytes could be initiated by oxidative stress occurring in the process of oocytes isolation. This is supported by the fact that initial phosphorylation of Ser473 in oocytes declined when fresh porcine OCCs were cultured for $5 \mathrm{~h}$ in gonadotropin-free medium and deprived of cumulus before western blot analysis. Ser473 was rephosphorylated in $3 \mathrm{~h}$ when OCCs were cultured in $\mathrm{FSH} / \mathrm{LH}$-supplemented medium subsequently (J Kalous, M Kubelka \& J Motlík 2007, unpublished data). After porcine oocytes were pretreated with dbcAMP for $20 \mathrm{~h}$, both Ser473 and Thr308 residues were nonphosphorylated. This is in accordance with the data on somatic cells, indicating that enhanced level of cAMP and elevated activity of PKA have inhibitory effects on the PI3K/PDK/AKT signaling and suppress phosphorylation of AKT on both Thr308 and Ser473 (Kim et al. 2001, Nijholt et al. 2008).

When DOs were cultured in control conditions, phosphorylation of the Ser473 residue and activity of AKT were elevated at the time of GVBD, indicating that AKT is possibly implicated in the regulation of events leading to GVBD. It has been published that separate phosphorylation of AKT on either Thr308 or Ser473 was sufficient to induce AKT activation (Lengyel et al. 2004, Viniegra et al. 2005). Nevertheless, phosphorylation of AKT on both Thr308 and Ser473 is a prerequisite for full activation of AKT (Alessi et al. 1996). Moreover, phosphorylation of AKT on Ser473 represents an essential prerequisite for autophosphorylation of Thr308 (Goren et al. 2008). Also, our data on porcine DOs reveal that increase in Thr308 phosphorylation occurred later than Ser473 phosphorylation. In cultured porcine DOs, phosphorylation of Thr308 was postponed until GVBD and reached maximal intensity at the
LD/MI-stage, implying that phosphorylation of Thr308 is important to support the activity of AKT during the MI-spindle formation. However, the ratio of Ser473 and Thr308 phosphorylation necessary for full AKT activation is not known. The results presented reveal that the time course of AKT phosphorylation and activation was somewhat different in samples of dbcAMP-nontreated and dbcAMP-pretreated oocytes, as the dbcAMP pretreatment resulted in a more synchronous progression of meiosis during subsequent control culture. It can be proposed that phosphorylated Ser473 and Thr308 act individually in the regulation of meiosis in porcine oocytes. Also, the data on mouse oocytes suggest that AKT phosphorylated on either Ser473 or Thr308 residue is separately distributed in oocytes and plays different roles in the regulation of meiosis (Kalous et al. 2006, Hoshino \& Sato 2008).

The phosphatidylinositol analog $\mathrm{SH}-6$ has been characterized as an effective AKT inhibitor that decreases AKT activity and induces apoptosis in cancer cells (Kozikowski et al. 2003). GVBD was not prevented when mouse and cattle oocytes were treated with $\mathrm{SH}-6$; however, progression of meiosis was affected in treated oocytes (Tomek \& Smiljakovic 2005, Hoshino \& Sato 2008). Also our experiments revealed that GVBD was not inhibited in SH-6-treated porcine DOs. Surprisingly, we observed that resumption of meiosis was accelerated during the $\mathrm{SH}-6$ treatment. It has been shown that PKA activation leads to inhibition of the PI3K/PDK/AKT signaling and reduction in AKT phosphorylation (Kim et al. 2001, Nijholt et al. 2008). However, phosphatidylinositol analogs like $\mathrm{SH}-6$, employed to inhibit AKT, also negatively affect PKA activity (Gills et al. 2007). It is possible to deduce that during $\mathrm{SH}-6$ treatment of porcine DOs, a reduction in PKA activity was accelerated, the activity of PKA substrates including PI3K/AKT and WEE1 kinase was affected, and hence GVBD promoted. Upon these conditions, the direct inhibitory effect of $\mathrm{SH}-6$ on AKT was counteracted by the $\mathrm{SH}-6$-induced suppression of PKA. Consequently, suppressed PKA activity promoted phosphorylation of AKT during resumption of meiosis and hence interfered with AKT inhibition in $\mathrm{SH}$ 6-treated DOs. Suppression of AKT by SH-6 became apparent after GVBD, when Thr308 phosphorylation and activity of AKT decreased; however, phosphorylation of Ser473 was not affected. The data reporting that phosphorylation of AKT on either Ser473 or Thr308 is regulated by different upstream mechanisms (Stokoe et al. 1997, Sarbassov et al. 2005) give explanation of the fact that phosphorylation of Ser473 in porcine DOs was not influenced by SH-6.

Our earlier data indicate that resumption of meiosis in porcine oocytes is accompanied by a substantial increase in CDK1 and MAP kinase activities (Kalous et al. 1993, Kubelka et al. 1995). The data presented here reveal that reduction in AKT activity occurring during SH-6 treatment did not prevent initial activation of CDK1 
and MAP kinase essential for induction of GVBD. However, when GVBD was accomplished, both CDK1 and MAP kinase were inhibited in $\mathrm{SH}$-6-treated porcine DOs. In starfish oocytes, AKT downregulates MYT1, a regulatory kinase of CDK1 and hence induces the initial activation of cyclin B/CDK1 at the meiotic G2/M-phase transition (Okumura et al. 2002). Our results indicate that in SH-6-treated porcine DOs, induction of GVBD and MPF activation is either AKT-independent or reduced activity of AKT is sufficient to support resumption of meiosis. We observed that in control culture, AKT became phosphorylated at Thr308 around GVBD. In contrast, in SH-6-treated DOs, phosphorylation of Thr308 was reduced, and progression of meiosis was arrested in early MI-stage. These data indicate that phosphorylation of AKT at Thr308 is not implicated in the events leading to the resumption of meiosis in porcine DOs; however, a full phosphorylation of the Thr308 is likely to be essential for meiosis to progress to the MI- and MII-stage. Similarly, inhibition of AKT in cattle oocytes did not prevent GVBD; however, meiosis was arrested in the MI-stage (Tomek \& Smiljakovic 2005).

In mouse MI- and MII-stage oocytes, AKT phosphorylated at Ser473 was localized with microtubules, while Thr308-phosphorylated AKT was present in pericentriolar material (Hoshino et al. 2004). Activated AKT phosphorylated at Thr308 is able to phosphorylate and inhibit GSK3 during mitosis (Cross et al. 1995, Cohen \& Frame 2001). Such AKT-mediated phosphorylation and activation of GSK3 occur on the centrosome and spindle poles and is involved in stabilization of microtubules in this region (Wakefield et al. 2002). We suggest that suppression of activity of AKT in porcine DOs induced by $\mathrm{SH}-6$ led to an increase of GSK3 activity in centrosomal region. Consequently, progress of meiosis was interrupted, as assembly of Ml-spindles was disturbed and aberrant Ml-spindles were formed. In conclusion, our results on porcine oocytes indicate that AKT is involved in the regulation of CDK1 activity during meiosis, and activated AKT is necessary for successful course of meiotic maturation.

\section{Materials and Methods}

\section{Experimental design}

The aim of the present study was to investigate the role of AKT in meiotic events occurring during in vitro maturation of porcine oocytes. To avoid the necessity of inducing meiotic in vitro progression in OCCs by gonadotropins and thus influence AKT pathway in oocytes by hormonal stimulus, the study was performed on DOs maturing in gonadotropin-free medium.

In experiment 1, phosphorylation and activation of AKT during control culture of dbcAMP-nontreated porcine DOs were investigated. Fresh OCCs were stripped of cumulus before culture, and DOs were further cultured in control medium for 3, $5,10,15,24$, and $44 \mathrm{~h}$. After culture, DOs were proceeded for western blot analysis, kinase assays, and assessment of nuclear maturation. In experiment 2, the effect of AKT inhibitor $\mathrm{SH}-6$ upon AKT activity and meiotic progression in DOs were analyzed. To achieve more synchronous progression of meiosis, porcine OCCs were pretreated with $1 \mathrm{mM}$ dbcAMP for $20 \mathrm{~h}$, and OCCs were deprived of cumulus subsequently. The DOs were further cultured either in control or SH-6 $(50 \mu \mathrm{M})$ supplemented medium for 5, 10, 15, 24, and $44 \mathrm{~h}$. After culture, DOs were subjected to phase-contrast and immunofluorescent microscopy, western blot analysis, and kinase assays.

\section{Oocyte collection, culture, and evaluation}

Ovaries, collected from slaughtered noncycling gilts, were transported to the laboratory in physiological saline at $20^{\circ} \mathrm{C}$. The ovaries were briefly washed in physiological saline, and OCCs were aspirated from 2- to 5-mm follicles using a 21-gauge needle attached to a syringe. M-199 medium (Sigma) supplemented with 5\% BSA (Sigma), $0.68 \mathrm{mM}$ glutamine, $100 \mathrm{mM}$ sodium pyruvate, $200 \mathrm{mM}$ cysteamine, $100 \mathrm{IU} / \mathrm{ml}$ penicillin, and $0.1 \mathrm{mg} / \mathrm{ml}$ streptomycin (Sigma) was used as a control medium. In experiment 1, the OCCs were deprived of cumulus before culture, and DOs were further cultured in nonsupplemented control medium. In experiment 2, the OCCs were precultured for $20 \mathrm{~h}$ in control medium supplemented with dbcAMP (1 mM final concentration; Calbiochem, San Diego, CA, USA). OCCs were stripped of cumulus after preculture, and DOs were further cultured either in nonsupplemented control medium or in control medium supplemented with SH-6 (50 $\mu \mathrm{M}$ final concentration; Calbiochem) for defined time intervals. All cultures were performed in a humidified atmosphere of $5 \% \mathrm{CO}_{2}$ at $38.5{ }^{\circ} \mathrm{C}$. At the end of culture, the DOs were washed in physiological saline, and after the last wash, the oocytes were fixed for microscopy or stored at $-80{ }^{\circ} \mathrm{C}$ until western blot or kinase assay analysis.

\section{Western blotting}

Oocytes were lysed in $10 \mu \mathrm{l}$ SDS/PAGE sample buffer (62.5 mM Tris/ $\mathrm{HCl}, \mathrm{pH} 6.8$, at $25^{\circ} \mathrm{C}, 2 \% \mathrm{w} / \mathrm{v}$ SDS, $10 \%$ $\mathrm{v} / \mathrm{v}$ glycerol, $0.01 \% \mathrm{w} / \mathrm{v}$ bromophenol blue, and $5 \% \mathrm{v} / \mathrm{v}$ 2-mercaptoethanol). Samples were heated at $100{ }^{\circ} \mathrm{C}$ for $3 \mathrm{~min}$ and subjected to SDS/PAGE. After electrophoresis, proteins were electrically transferred onto PVDF membrane (Immobilon-P; Millipore, Bedford, MA, USA) using a semi-dry blotting system. Subsequently, blotted membranes were blocked for $1 \mathrm{~h}$ in $5 \%(\mathrm{v} / \mathrm{v})$ nonfat milk (Sigma) in T-TBS (Tris/HCl-buffered saline ( $\mathrm{pH} 7.6)$ with $0.05 \%$ Tween 20$)$. T-TBS was used for all following incubations and washes. Blocked membranes were incubated overnight at $4{ }^{\circ} \mathrm{C}$ with a PKB-specific primary antibody diluted 1:1000 in T-TBS with 5\% nonfat milk (pAKT Ser473 (SC-7985), pAKT Thr308 (SC-16646), or AKT1 antibody (SC-5298)); all primary antibodies were purchased from Santa Cruz Biotechnology (Santa Cruz, CA, USA). The blots were then washed (thrice for $5 \mathrm{~min}$ ) in T-TBS and incubated for $1 \mathrm{~h}$ at room temperature $\left(18-25^{\circ} \mathrm{C}\right)$ with HRP-linked donkey anti-rabbit IgG (Amersham; 1:10 000, phospho-AKT detection) or rabbit antimouse IgG (Amersham; 1:15 000, total AKT protein detection), both diluted in T-TBS with 5\% nonfat milk. After extensive washing (three to five times for $5 \mathrm{~min}$ ), the immunoreactive signal was detected using ECL (Amersham International). 


\section{Histone $\mathrm{H} 1$ assay}

At each time interval, ten oocytes/sample were collected, washed $(4 \times)$ in PBS, and transferred into $3 \mu \mathrm{PBS}$ in Eppendorf tubes. Samples were immediately frozen on solid $\mathrm{CO}_{2}$ and stored at $-80{ }^{\circ} \mathrm{C}$ until assays were performed. The histone $\mathrm{H} 1$ and myelin basic protein (MBP) kinase activities were measured as previously described (Motlik et al. 1998); $\mathrm{H} 1$ and MBP are substrates for CDK1 and MAPK respectively. Data from autoradiograms were analyzed using Scion Image software (Scion Corporation, Frederick, MD, USA).

\section{AKT kinase assay}

Eppendorf tubes with frozen oocytes (200 DOs in each tube) were placed on ice, and $200 \mu \mathrm{l} 1 \times$ ice-cold cell lysis buffer (catalog no. 9803; Cell Signaling Technology, Beverly, MA, USA) containing $1 \mathrm{mM}$ phenylmethylsulphonyl fluoride was added to each tube; oocytes were lysed by several cycles of freezing and thawing. After centrifugation (14000 $\mathrm{g}$ for $10 \mathrm{~min}$ at $4{ }^{\circ} \mathrm{C}$ ), the supernatant was removed and $3 \mu$ AKT1 MAB (SC-5298; Santa Cruz Biotechnology) was added, and then the samples were incubated for $1 \mathrm{~h}$ at $4{ }^{\circ} \mathrm{C}$. Protein A Sepharose (Sigma) $(200 \mu \mathrm{l}$ of the suspension) was added, and the mixture was incubated at $4{ }^{\circ} \mathrm{C}$ overnight. The pellet was collected by low-speed centrifugation and washed twice with ice-cold cell lysis buffer (catalog no. 9803; Cell Signaling Technology) and ice-cold kinase buffer (catalog no. 9802; Cell Signaling Technology). The washed pellet was assayed for PKB activity as follows: the reaction was started by adding $30 \mu \mathrm{l}$ kinase buffer $(25 \mathrm{mM}$ Tris/ $\mathrm{HCl}, \mathrm{pH} 7.5,5 \mathrm{mM}$ $\beta$-glycerophosphate, $2 \mathrm{mM}$ dithiothreitol, $0.1 \mathrm{mM} \mathrm{Na} \mathrm{VO}_{4}$, $10 \mathrm{mM} \mathrm{MgCl}_{2}$, and $200 \mu \mathrm{M}$ ATP) to each sample together with $2 \mu \mathrm{g}$ GSK3 fusion protein (Cell Signaling Technology) as an external substrate for AKT and $500 \mu \mathrm{Ci} / \mathrm{ml}\left[\gamma_{-}{ }^{32} \mathrm{P}\right]$ ATP $(10 \mathrm{mCi} / \mathrm{ml}$; Amersham). The reaction was performed for $30 \mathrm{~min}$ at $30{ }^{\circ} \mathrm{C}$ and terminated by adding $30 \mu \mathrm{l} 4 \times$ concentrated SDS/PAGE sample buffer and boiled for $3 \mathrm{~min}$. After SDS/PAGE (15\% w/v PAGE) (Laemmli 1970), the gels were stained with Coomassie Blue R250 (Serva, Heidelberg, Germany), destained overnight, dried, and autoradiographed. Subsequently, the gel was scanned with a Fujifilm BAS-2500 Phosphoimager, and the data were analyzed by Scion Image software.

\section{Morphological analyses of oocytes}

To check the current stage of maturation at the time of sample collection, an aliquot of 30-40 DOs was mounted on microscope slides with Vaseline (Sigma) strips, covered with cover glass, and fixed in solution ethanol:acetic acid (3:1 v/v) for $48 \mathrm{~h}$. Staining was performed with $2 \%$ orcein in $50 \%$ aqueous acetic acid and $1 \%$ sodium citrate. The slides were then placed in $40 \%$ acetic acid and observed with a phasecontrast NU Zeiss microscope.

\section{Immunocytochemistry}

Denuded zona-intact oocytes were washed in PBS, fixed for $1 \mathrm{~h}$ in $3.7 \%$ paraformaldehyde (16005, Aldrich), washed in PBS, and stored in PBS with 1\% sodium azide. Such prepared oocytes were washed for 30 min in PBS containing 1\% Triton-X (T9284, Sigma-Aldrich) and subsequently blocked in $2 \%$ BSA fraction $\mathrm{V}$ (Sigma) for $2 \mathrm{~h}$ in PBS. The oocytes were incubated overnight at $4{ }^{\circ} \mathrm{C}$ with mouse monoclonal anti-acetylated tubulin antibody (T7451, Sigma, diluted 1:500). The excess primary antibody was removed by extensive washing in PBS containing $0.2 \%$ BSA $/ 0.05 \%$ saponin (16109, Sigma). Oocytes were then incubated with donkey anti-mouse antibody conjugated with Alexa Flour-594 (Invitrogen, diluted 1:800) in PBS/0.2\% $\mathrm{BSA} / 0.05 \%$ saponin for $60 \mathrm{~min}$ at room temperature. As a control, oocytes were incubated only with secondary antibody. The well-washed oocytes were mounted in mounting medium with DAPI ( $\mathrm{H}-1500$; Vector Laboratories, Peterborough, UK) and examined using confocal microscopy (Leica).

\section{Statistical analyses}

Statistical analysis of all data was performed using NCSS 2000 computer software (NCSS, Kaysville, UT, USA). Error sections in graphs express confidence limits. The frequencies of meiosis progression were pooled from three replicate experiments, and cumulative results were subjected to $\chi^{2}$ analysis. Differences were considered significant at $P<0.05$. A total of 2634 oocytes were subjected to morphological analyses. The results of the western blotting and AKT kinase assays were quantified by Scion Image software and expressed as the means \pm s.D. of three independent experiments. The data were normalized to the mean value by mean centering.

\section{Declaration of interest}

The authors declare that there is no conflict of interest that would prejudice the impartiality of this scientific work.

\section{Funding}

This work was supported by the Academy of Sciences of the Czech Republic (program IRP IAPG No. AV0Z50450515) and Grant Agency of the Czech Republic (grant numbers 204/06/1297, 524/07/1087, 523/03/0857).

\section{References}

Alessi DR, Andjelkovic M, Caudwell B, Cron P, Morrice N, Cohen P \& Hemmings BA 1996 Mechanism of activation of protein kinase B by insulin and IGF-I. EMBO Journal 15 6541-6551.

Andersen CB, Sakaue H, Nedachi T, Kovacina KS, Clayberger C, Conti M \& Roth RA 2003 Protein kinase B/Akt is essential for the insulin- but not progesterone-stimulated resumption of meiosis in Xenopus oocytes. Biochemical Journal 369 227-238.

Baldin V, Theis-Febvre N, Benne C, Froment C, Cazales M, Burlet-Schiltz O \& Ducommun B 2003 PKB/Akt phosphorylates the CDC25B phosphatase and regulates its intracellular localization. Biology of the Cell 95 547-554.

Bornslaeger EA \& Schultz RM 1985 Regulation of mouse oocyte maturation: effect of elevating cumulus cell cAMP on oocyte cAMP levels. Biology of Reproduction 33 698-704.

Cohen P \& Frame S 2001 The renaissance of GSK3. Nature Reviews. Molecular Cell Biology 2 769-776.

Coleman TR \& Dunphy WG 1994 Cdc2 regulatory factors. Current Opinion in Cell Biology 6 877-882. 
Conti M 2002 Specificity of the cyclic adenosine $3^{\prime}, 5^{\prime}$-monophosphate signal in granulose cell function. Biology of Reproduction 67 1653-1661.

Conti M, Andersen CB, Richard FJ, Sitsukawa K \& Tsafriri A 1998 Role of cyclic nucleotide phosphodiesterases in resumption of meiosis. Molecular and Cellular Endocrinology 145 9-14.

Cross DA, Alessi DR, Cohen P, Andjelkovich M \& Hemmings BA 1995 Inhibition of glycogen synthase kinase-3 by insulin mediated by protein kinase B. Nature 378 785-789.

DeuterReinhardt M, Apell G, Pot D, Klippel A, Williams LT \& Kavanaugh WM 1997 SIP/SHIP inhibits Xenopus oocyte maturation induced by insulin and phosphatidylinositol 3-kinase. Molecular and Cellular Biology 17 2559-2565.

Duckworth BC, Weaver JS \& Ruderman JV 2002 G2 arrest in Xenopus oocytes depends on phosphorylation of cdc25 by protein kinase A. PNAS 99 16794-16799.

Funahashi H, Cantley TC \& Day BN 1997 Synchronization of meiosis in porcine oocytes by exposure to dibutyryl cyclic adenosine monophosphate improves developmental competence following in vitro fertilization. Biology of Reproduction 57 49-53.

Gautier J \& Maller JL 1991 Cyclin B in Xenopus oocytes: implications for the mechanism of pre-MPF activation. EMBO Journal 10 177-182.

Gills JJ, Castillo SS, Zhang Ch, Petukhov PA, Memmott RM, Hollingshead M, Warfel N, Han J, Kozikowski AP \& Dennis PA 2007 Phosphatidylinositol ether lipid analogues that inhibit AKT also independently activate the stress kinase, p38, through MKK3/6-independent and -dependent mechanisms. Journal of Biological Chemistry 282 27020-27029.

Goren I, Müller E, Pfeischifter J \& Frank S 2008 Thr308 determines Akt1 localization in insulin-stimulated keratinocites. Biochemical and Biophysical Research Communications 372 103-107.

Han SJ \& Conti M 2006 New pathways from PKA to the Cdc2/cyclin B complex in oocytes: Wee1B as a potential PKA substrate. Cell Cycle $\mathbf{5}$ 227-231.

Han SJ, Chen R, Paronetto MP \& Conti M 2005 Wee1B is an oocyte-specific kinase involved in the control of meiotic arrest in the mouse. Current Biology 15 1670-1676.

Han SJ, Vaccari S, Nedachi T, Andersen CB, Kovacina KS, Roth RA \& Conti M 2006 Protein kinase B/Akt phosphorylation of PDE3A and its role in mammalian oocyte maturation. $E M B O$ Journal 25 5716-5725.

Hehl S, Stoyanov B, Oehrl W, Schonnherr R, Wetzker R \& Heinemann SF 2001 Phosphoinositide 3-kinase-C induces Xenopus oocyte maturation via lipid kinase activity. Biochemical Journal 360 691-698.

Hoshino Y \& Sato E 2008 Protein kinase B (PKB/Akt) is required for the completion of meiosis in mouse oocytes. Developmental Biology 314 215-223.

Hoshino Y, Yokoo M, Yoshida N, Sasada H, Matsumoto H \& Sato E 2004 Phosphatidylinositol 3-kinase and Akt participate in the FSH-induced meiotic maturation of mouse oocytes. Molecular Reproduction and Development 69 77-86.

Jessus C \& Beach D 1992 Oscillation of MPF is accompanied by periodic association between cdc25 and cdc2-cyclin B. Cell 68 323-332.

Kalous J, Kubelka M, Rimkevicova Z, Guerrier P \& Motlik J 1993 Okadaic acid accelerates germinal vesicle breakdown and overcomes cycloheximide- and 6-dimethylaminopurine block in cattle and pig oocytes. Developmental Biology 157 448-454.

Kalous J, Solc P, Baran V, Kubelka M, Schultz RM \& Motlik J 2006 PKB/Akt is involved in resumption of meiosis in mouse oocytes. Biology of the Cell 98 111-123.

Kandel ES, Skeen J, Majewski N, Di Cristofano A, Pandolfi PP \& Feliciano CS 2002 Activation of Akt/protein kinase B overcomes a $\mathrm{G}(2) / \mathrm{M}$ cell cycle checkpoint induced by DNA damage. Molecular and Cellular Biology 22 7831-7841.

Katayama K, Fujita N \& Tsuruo T 2005 Akt/protein kinase B-dependent phosphorylation and inactivation of WEE1Hu promote cell cycle progression at G2/M transition. Molecular and Cellular Biology 25 5725-5737.

Kim NH \& Menino AR 1995 Effects of stimulators of protein kinases A and C and modulators of phosphorylation on plasminogen activator activity in porcine oocyte-cumulus cell complexes during in vitro maturation. Molecular Reproduction and Development 40 364-370.
Kim S, Jee K, Kim D, Koh H \& Chung J 2001 Cyclic AMP inhibits Akt activity by blocking the membrane localization of PDK1. Journal of Biological Chemistry 276 12864-12870.

Konishi H, Matsuzaki H, Tanaka M, Takemura Y, Kuroda S, Ono Y \& Kikkawa U 1997 Activation of protein kinase B (Akt/RAC-protein kinase) by cellular stress and its association with heat shock protein Hsp27. FEBS Letters 410 493-498.

Kovo M, Kandli-Cohen M, Ben-Hamin M, Galiani D, Carr DW \& Dekel N 2006 An active protein kinase A (PKA) is involved in meiotic arrest of rat growing oocytes. Reproduction 132 33-43.

Kozikowski AP, Sun H, Brognard J \& Dennis PA 2003 Novel PI analogues selectively block activation of the pro-survival serine/ threonine kinase Akt. Journal of the American Chemical Society $\mathbf{1 2 5}$ 1144-1145.

Kubelka M, Rimkevicova Z, Guerrier P \& Motlik J 1995 Inhibition of protein synthesis affects histone $\mathrm{HI}$ kinase, but not chromosome condensation activity, during the first meiotic division of pig oocytes. Molecular Reproduction and Development 4 63-69.

Laemmli UK 1970 Cleavage of structural proteins during the assembly of the head of bacteriophage T4. Nature 15 680-685.

Lengyel F, Vertes Z, Kovacs KA, Kornyei JL, Sumegi B \& Vertes M 2004 Expression and activation of Akt/protein kinase B in sexually immature and mature rat uterus. Journal of Steroid Biochemistry and Molecular Biology 91 285-288.

Liu XJ, Sorisky A, Zhu L \& Pawson T 1995 Molecular cloning of an amphibian insulin receptor substrate 1-like CDNA and involvement of phosphatidylinositol 3-kinase in insulin-induced Xenopus oocyte maturation. Molecular and Cellular Biology 15 3563-3570.

Maehama T, Taylor GS \& Dixon JE 2001 PTEN and myotubularin: novel phosphoinositide phosphatases. Annual Review of Biochemistry 70 247-279.

Masciarelli S, Horner K, Liu C, Park SH, Hinckley M, Hockmam S, Nedachi T, Jin C, Conti M \& Manganiello V 2004 Cyclic nucleotide phosphodiesterase 3A-deficient mice as a model of female infertility. Journal of Clinical Investigation 114 196-205.

Moreno FJ \& Avila J 1998 Phosphorylation of stathmin modulates its function as a microtubule depolymerizing factor. Molecular and Cellular Biochemistry 183 201-209.

Motlik J, Pavlok A, Kubelka M, Kalous J \& Kalab P 1998 Interplay between CDC2 kinase and MAP kinase pathway during maturation of mammalian oocytes. Theriogenology 49 461-469.

Nemcova L, Nagyova E, Petlach M, Tomanek M \& Prochazka R 2007 Molecular mechanisms of insulin-like growth factor 1 promoted synthesis and retention of hyaluronic acid in porcine oocyte-cumulus complexes. Biology of Reproduction 76 1016-1024.

Nijholt IM, Dolga AM, Ostroveanu A, Luiten PG, Schmidt M \& Eisel UL 2008 Neuronal AKAP150 coordinates PKA and Epac-mediated PKB/Akt phosphorylation. Cellular Signalling 20 1715-1724.

Nilsson I \& Hoffmann I 2000 Cell cycle regulation by the Cdc25 phosphatase family. Progress in Cell Cycle Research 4 107-114.

Okumura E, Fukuhara T, Yoshida H, Hanada S, Kozutsumi R, Mori M, Tachibana K \& Kishimoto T 2002 Akt inhibits Myt1 in the signaling pathway that leads to meiotic G2/M transition. Nature Cell Biology $\mathbf{4}$ $111-116$.

Roberts EC, Shapiro PS, Nahreini TS, Pages G, Pouyssegur J \& Ahn NG 2002 Distinct cell cycle timing requirements for extracellular signal-regulated kinase and phosphoinositide 3-kinase signaling pathways in somatic cell mitosis. Molecular and Cellular Biology 22 7226-7241.

Sarbassov DD, Guertin DA, Ali SM \& Sabatini DM 2005 Phosphorylation and regulation of Akt/PKB by the rictor-mTOR complex. Science $\mathbf{3 0 7}$ 1098-1101.

Shibuya EK 2003 G2 cell cycle arrest - a direct link between PKA and Cdc25C. Cell Cycle 2 39-41.

Shtivelman E 2003 Promotion of mitosis by activated protein kinase B after DNA damage involves Polo-like kinase 1 and checkpoint protein CHFR. Molecular Cancer Research 1 959-969.

Shtivelman E, Sussman J \& Stokoe D 2002 A role for Pl 3-kinase and PKB activity in the G2/M phase of the cell cycle. Current Biology 12 919-924. 
Smith G \& Ding J 2005 Insulin signaling through glycogen synthase kinase3 regulates chromatin condensation and segregation in mouse oocytes. Proceedings of the Society for the Study of Reproduction, 38th Annual Meeting, Quebec City, Quebec, Canada. Abstract M394.

Stokoe D, Stephens LR, Copeland T, Gaffney PR, Reese CB, Painter GF, Holmes AB, McCormick F \& Hawkins PT 1997 Dual role of phosphatidylinositol-3,4,5-trisphosphate in the activation of protein kinase B. Science 277 567-570.

Tomek W \& Smiljakovic T 2005 Activation of Akt (protein kinase B) stimulates metaphase I to metaphase II transition in bovine oocytes. Reproduction 130 423-430.

Tsafriri A, Chun SY, Zhang R, Hsueh AJ \& Conti M 1996 Oocyte maturation involves compartmentalization and opposing changes of cAMP levels in follicular somatic and germ cells: studies using selective phosphodiesterase inhibitors. Developmental Biology 178 393-402.

Vanhoutte L, Nogueira D, Gerris J, Dhont M \& De Sutter P 2008 Effect of temporary nuclear arrest by phosphodiesterase 3-inhibitor on morphological and functional aspects of in vitro matured mouse oocytes. Molecular Reproduction and Development 75 1021-1030.
Viniegra JG, Martınez N, Modirassari P, Losa JH, Parada Cobo C, Lobo VK, Luquero CI, Alvarez-Vallina L, Ramon y Cajal S, Rojas JM et al. 2005 Full activation of $\mathrm{PKB} / \mathrm{Akt}$ in response to insulin or ionizing radiation is mediated through ATM. Journal of Biological Chemistry $2804029-4036$.

Wakefield JG, Stephens DJ \& Tavaré JM 2002 A role of glycogen synthase kinase-3 in mitotic spindle dynamics and chromosome alignment. Journal of Cell Science 116 637-646.

Wang X, Liu X, Dunn R, Ohl D \& Smith G 2003 Glycogen synthase kinase-3 regulates mouse oocyte homologue segregation. Molecular Reproduction and Development 64 96-105.

Received 5 May 2008

First decision 9 December 2008

Revised manuscript received 6 July 2009

Accepted 24 July 2009 